\section{Targeted DNA transfection into the mouse central nervous system using laser- induced stress waves}

\author{
Yasushi Satoh* \\ National Defense Medical College \\ Department of Biochemistry I \\ Tokorozawa 359-8513, Japan
}

\section{Yasunari Kanda}

National Defense Medical College

Department of Pharmacology

Tokorozawa 359-8513, Japan

\section{Mitsuhiro Terakawa}

\section{Minoru Obara}

Keio University

Department of Electronics and Electrical Engineering

Kohoku-ku, Yokohama 223-8522, Japan

\author{
Katsushige Mizuno \\ Yasuhiro Watanabe \\ National Defense Medical College \\ Department of Pharmacology \\ Tokorozawa 359-8513, Japan
}

\section{Shogo Endo}

Okinawa Institute of Science and Technology

Unit for Molecular Neurobiology of Learning and Memory

JST, Gushikawa, 904-2234, Japan

\author{
Hidetoshi Ooigawa \\ Hiroshi Nawashiro \\ National Defense Medical College \\ Department of Neurosurgery \\ Tokorozawa 359-8513, Japan
}

\section{Shunichi Sato}

National Defense Medical College

Division of Biomedical Information Sciences

Tokorozawa 359-8513, Japan

\section{Kunio Takishima}

National Defense Medical College

Department of Biochemistry I

Tokorozawa 359-8513, Japan

\begin{abstract}
We investigated the feasibility of gene transfer into the mouse central nervous system (CNS) by applying nanosecond pulsed laser-induced stress waves (LISWs).
\end{abstract}

\footnotetext{
*Tel: +81-42-995-1373; Fax: +81-42-996-5189; E-mail: ys@ndmc.ac.jp
}

Intraventricular or hippocampal injection of a reporter gene [enhanced green fluorescent protein (EGFP)] followed by application of LISWs showed this method to be efficient in the CNS of newborn and adult mice. Cells expressing EGFP reside at least $3.5 \mathrm{~mm}$ from the surface of the tissue, while no apparent damage was detected. Additionally, expression of EGFP was limited to the area that was exposed to LISWs. Using this method, the formulation of plasmid DNA by cationic transfer reagent polyethylenimine proved to be effective for improving transfer efficiency into the CNS. () 2005 Society of Photo-Optical Instrumentation Engineers. [DOI: 10.1117/1.2128432]

Keywords: laser-induced stress wave; central nervous system; stereotaxic injection; in vivo transfection; polyethylenimine.

Paper 05201LR received Jul. 20, 2005; revised manuscript received Aug. 29, 2005; accepted for publication Sep. 15, 2005; published online Nov. 23, 2005.

\section{Introduction}

Gene transfer into the central nervous system (CNS) offers a large number of techniques for exploring the function of neural systems and a potential basis for therapy of neurological diseases. There still remain insurmountable difficulties in neuronal tissue transfection, which impede the application of molecular approaches to neurobiology of the CNS. One approach for the delivery of nucleic acids into specific regions in the CNS is the use of recombinant viral vectors. ${ }^{1,2}$ Generally, these methods are the most efficient for CNS tissue, but some problems such as assurance of safety and the immune response to viral vectors remain to be solved before application in human gene therapy. Various physical methods such as electroporation $^{3}$ and ultrasound ${ }^{4}$ have also been investigated, but problems associated with low transfer efficiency and limited targeting characteristics in the CNS remain.

There has been much interest in the use of lasers for nonviral targeted gene transfer because of the high spatial controllability of laser energy. However, most reports on lasermediated gene transfer have dealt with single-cell-basis transfection by direct irradiation of the cell membrane with a focused laser beam, ${ }^{5}$ and therefore this approach cannot be applied to deep tissue because of limited optical penetration. Another problem is the limited diffusibility of plasmid DNA in the brain, which may hinder the gene delivery into the deep tissue. We have previously applied laser induced stress waves (LISWs) to gene transfer into rat skin ${ }^{6}$ and NIH $3 \mathrm{~T} 3$ cells. $^{7}$ These reports indicated that highly-efficient and site-specific gene transfer can be achieved by this method, and that a LISW can propagate deeply from the surface of the tissue. Polyethylenimine (PEI) is a synthetic cationic vector and has been shown to be a versatile gene transfer tool in vivo. ${ }^{8}$ The plasmid DNA complexes formulated with $22 \mathrm{kDa}$ PEI in $5 \%$ glucose are sufficiently small and stable in physiological fluids to provide high diffusibility, ${ }^{8}$ although it seems unsatisfactory to obtain high transfection efficiency comparable with those of viral vectors.

In this study, we have combined the LISW method with DNA/PEI formulation to achieve effective gene transfer into the CNS, and this novel technique gives high spatial control-

1083-3668/2005/10(6)/060501/3/\$22.00 @ 2005 SPIE 


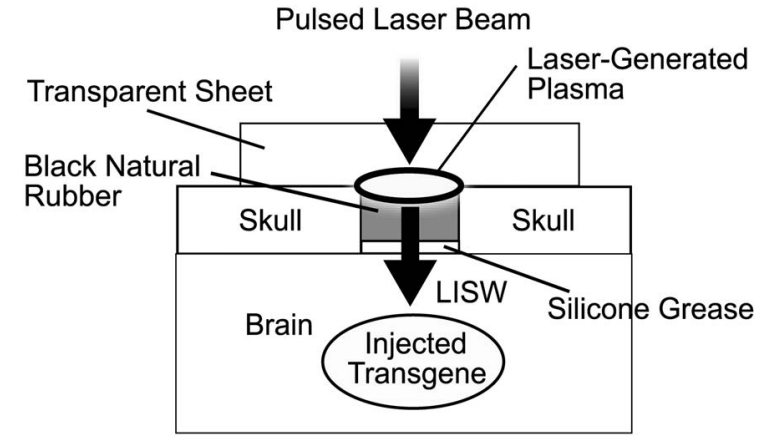

Fig. 1 Experimental arrangement for gene transfer into the CNS using a laser-induced stress wave (LISW).
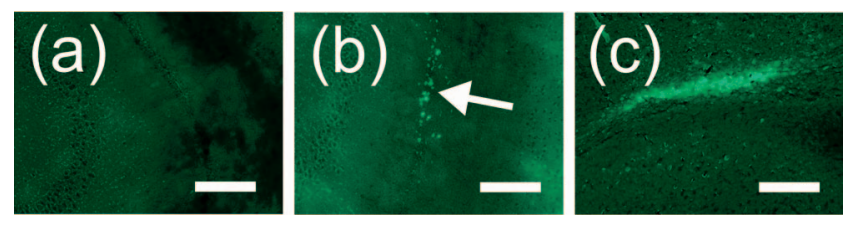

Fig. 2 (a) Sham-controlled operation on mice shows no fluorescence following a single intraventricular injection of the PEI alone (without DNA) and exposure to LISWs. (b) Without use of a LISW following a ventricular injection of DNA/PEI, limited expression of EGFP was detected. Arrow indicates the location of fluorescence induced above background autofluorescence. (c) Use of LISWs following a ventricular injection of DNA/PEI gives higher expression. Scale bars indicate $500 \mu \mathrm{m}$.

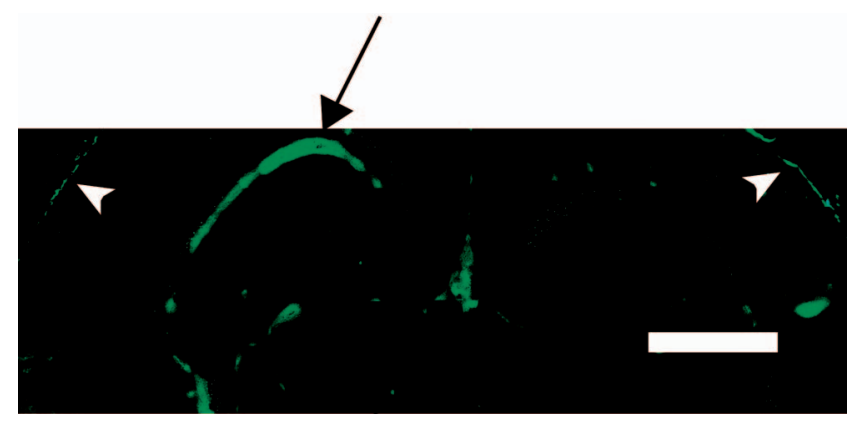

Fig. 4 Expression of EGFP detected in newborn mice. Arrow indicates the location that transgene was injected. Arrowheads indicate the surface of the brain. The scale bar indicates $1 \mathrm{~mm}$.

lability compared with viral vectors. Thus, this method is promising for conditional gene knockout in mice and may well be adaptable to clinical gene therapy in the CNS.

\section{Materials and Methods}

pLEGFP-N1, an expression plasmid for EGFP, was purchased from Clontech. The plasmid DNA solution was prepared according to the manufacturer's instructions using $3 \mu$ l of DNA and $6 \mu \mathrm{l}$ of Jet-PEI (PolyPlus-transfection) in 5\% glucose to obtain a PEI nitrogen/DNA phosphate $(\mathrm{N} / \mathrm{P})$ ratio of 5 . We used adult male C57BL6J mice (Japan SLC) weighing 28 to $32 \mathrm{~g}$. The studies were carried out according to the protocols approved by National Defense Medical College Board for Studies in Experimental Animals. Mice were anaesthetized with $50 \mathrm{mg} / \mathrm{kg}$ sodium pentobarbital and placed in a stereotaxic apparatus (SR-6N, Narishige). Stereotaxic injection was carried out as described previously. ${ }^{9}$ Briefly, the skull was
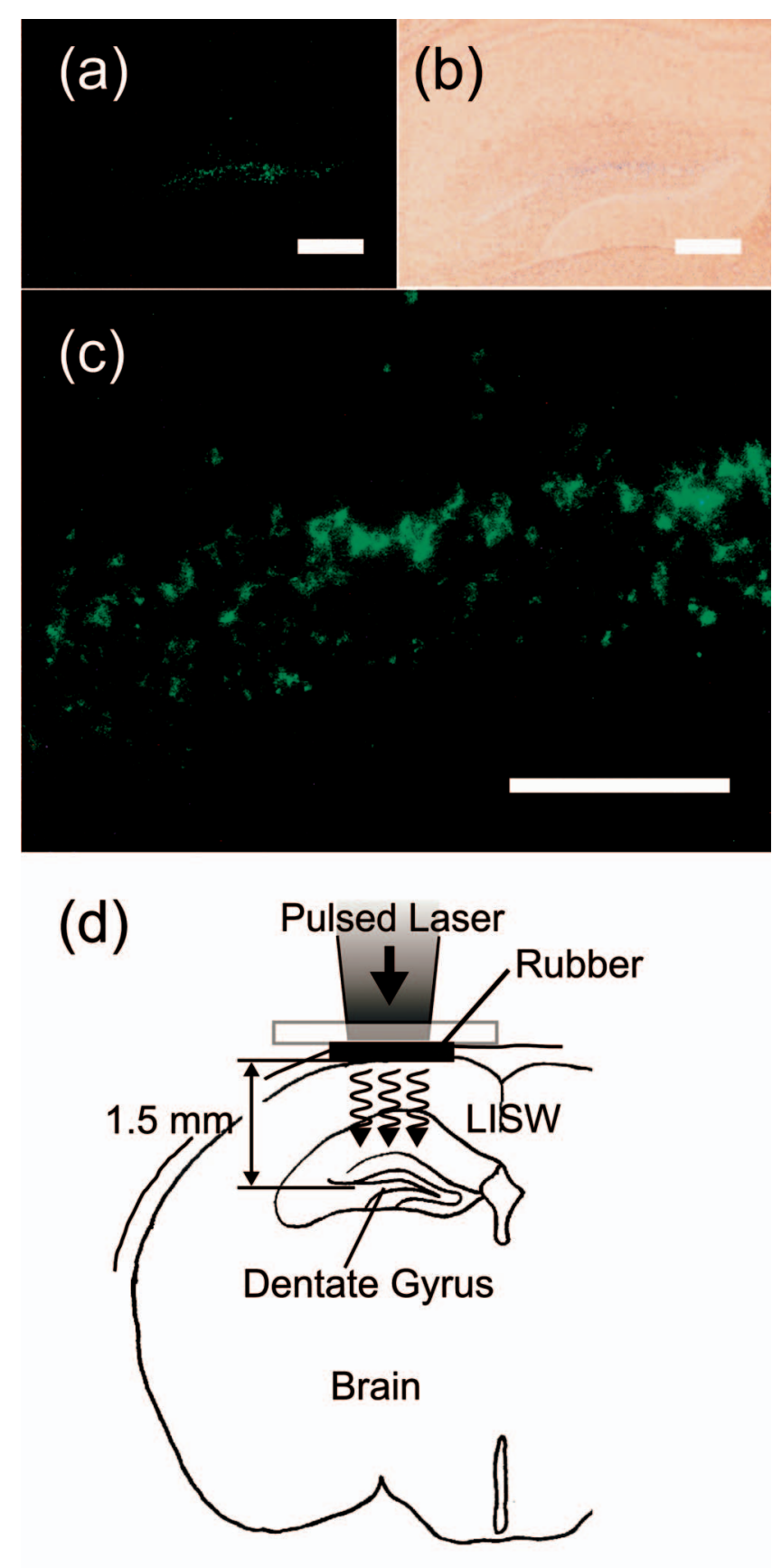

Fig. 3 (a) Expression of EGFP was detected when a gene was injected into the dentate gyrus. (b) A bright field image of the same region as shown in (a). (c) Higher magnification view of an area in (a). Arrows indicate the typical cells expressing EGFP. The scale bars indicate $200 \mu \mathrm{m}$ in (a, b) and $100 \mu \mathrm{m}$ in (c). (d) Schematic drawing of brain showing the location of sites that were sectioned relative to the site of LISW.

exposed, a small burr hole was drilled, and a Hamilton syringe with a 26-gauge needle was stereotaxically inserted into the lateral ventricle or hippocampus. The solution $(5 \mu \mathrm{l})$ was injected at a rate of $1 \mu \mathrm{l} / \mathrm{min}$. The needle was left in place for an additional $5 \mathrm{~min}$ to allow diffusion of the solution. For newborn mice, $2 \mu$ l of the solution was injected into the lateral ventricle after anaesthetization by hypothermia on ice. The experimental arrangement to generate a LISW was the 
same as described previously ${ }^{6}$ except for the diameter of the laser spot on the target (Fig. 1). A black natural rubber was placed as a laser target on the surface of brain. Silicone grease was used between the target and the surface to ensure acoustic impedance matching. On the rubber, a transparent polyethylene terephthalate sheet was adhered to confine the laserinduced plasma. After the injection, the nanosecond $(7.0 \mathrm{~ns}$, FWHM) pulsed laser light from the second harmonics $(532 \mathrm{~nm})$ of a Q-switched Nd:YAG laser was focused using an uncoated quartz lens $(f=200 \mathrm{~nm})$, and a spot of $1 \mathrm{~mm}$ in diameter on the target was irradiated to generate LISWs. The laser fluence was $1.9 \mathrm{~J} / \mathrm{cm}^{2}$ and three pulses of laser light were administered in all experiments. Each experiment was performed on a minimum of three animals. Mice were killed $72 \mathrm{~h}$ ( $24 \mathrm{~h}$ for newborn mice) after administration, fixed, and the brain was removed. Removed brains were sectioned with a cryostat microtome to $10 \mu \mathrm{m}$ in thickness as previously described. ${ }^{9}$ Expression of EGFP was histochemically examined with a fluorescent microscope (TE-2000E, Nikon). To investigate the depth profile of LISWs in tissue, pressure measurement was performed using a calibrated membrane-type hydrophone (H9C, Toray Engineering) for brain sections with various thicknesses. Sections were made on a vibrating microtome; to each section, a laser target was attached. The section was placed on the hydrophone membrane and the target was irradiated at a laser fluence of $1.9 \mathrm{~J} / \mathrm{cm}^{2}$ per pulse. Signals from the hydrophone were recorded in a high-frequency digital oscilloscope.

\section{Results}

The extent of brain damage caused by LISWs aiming at ventricle was examined at laser fluences of $0.4,1.0$, or $1.9 \mathrm{~J} / \mathrm{cm}^{2}$. The hematoxylin-eosin stained tissue sections from the irradiated brains showed no detectable damage (data not shown). Peak pressures of the LISWs at a laser fluence of $1.9 \mathrm{~J} / \mathrm{cm}^{2}$ were $74,35.2,31.5$, and $25.9 \mathrm{MPa}$ for brain section thicknesses of 0 (no section), 1, 2, and $3 \mathrm{~mm}$, respectively.

Administration of naked EGFP expression plasmids into one of the lateral ventricles of a mouse by use of LISWs showed limited EGFP expression at the site of injection whereas no expression was detected in tissue sections that were not exposed to LISW (data not shown). We then examined the effect of PEI on gene transfer efficiency with a LISW. Administration of DNA/PEI complex alone, without a LISW, showed some expression [Fig. 2(b)]. In contrast, relatively efficient and widespread expression of EGFP was observed when DNA/PEI complexes were injected into the tissue followed by exposure of LISWs [Fig. 2(c)]. Such expression was observed in cells adjacent to the ventricular space and only in the area that was exposed to LISWs. Sham-controlled operation after a single intraventricular injection of PEI without DNA shows no EGFP expression [Fig. 2(a)]. Figure 3(a) shows the expression of fluorescence following an injection of EGFP expression plasmids with PEI into the dentate gyrus of the adult mouse brain at a laser fluence of $1.9 \mathrm{~J} / \mathrm{cm}^{2}$. The bright field image of the same region is shown in Fig. 3(b) to identify the expression area in the dentate gyrus. A higher magnification view shows typical cells expressing fluorescence [Fig. 3(c)]. Cells expressing EGFP reside approxi- mately $1.5 \mathrm{~mm}$ deep below the target rubber located on the cortex surface [Fig. 3(d)]. Figure 4 shows expression of EGFP in newborn mice following an intraventricular injection into the left hemisphere. This sagittal section shows the expression of EGFP only in the left hemisphere. Cells expressing EGFP reside up to approximately $3.5 \mathrm{~mm}$ deep from the cortex surface located immediately beneath the irradiated spot. Note that almost no fluorescence is seen in the hippocampus of the right hemisphere. This result demonstrates that the LISW method is effective in transferring DNA into the cells of the developing nervous system.

\section{Discussion and Conclusion}

We proved that the procedure of administration using LISW with gene formulation by PEI is applicable to gene transfer into the CNS. Transgene expression using PEI was found in almost all the injected brains under the condition at a laser fluence at $1.9 \mathrm{~J} / \mathrm{cm}^{2}$, indicating high reproducibility of this method. Our method allowed efficient DNA delivery into cells several millimeters deep from the cortex surface. Because some target tissues in the CNS reside too deep to be treated with direct laser irradiation, LISW may provide a valuable method for a site-specific conditional knockout system in mice and for the treatment of inherited or acquired brain disease such as Alzheimer's disease or Parkinson's disease.

\section{Acknowledgments}

This work was partly supported by grants from the Japanese Ministry of Education, Science and Technology.

\section{References}

1. Y. Wang, L. A. Krushel, and G. M. Edelman, "Targeted DNA recombination in vivo using an adenovirus carrying the cre recombinase gene," Proc. Natl. Acad. Sci. U.S.A. 93, 3932-3936 (1996).

2. I. A. Franceschini, V. Feigenbaum-Lacombe, P. Casanova, M. LopezLastra, J. L. Darlix, and M. D. Dalcq, "Efficient gene transfer in mouse neural precursors with a bicistronic retroviral vector," J. Neurosci. Res. 65, 208-219 (2001).

3. S. Somiari, J. Glasspool-Malone, J. J. Drabick, R. A. Gilbert, R. Heller, M. J. Jaroszeski, and R. W. Malone, "Theory and in vivo application of electroporative gene delivery," Mol. Ther. 2, 178-187 (2000)

4. Y. Taniyama, K. Tachibana, K. Hiraoka, M. Aoki, S. Yamamoto, K. Matsumoto, T. Nakamura, T. Ogihara, Y. Kaneda, and R. Morishita, "Development of safe and efficient novel nonviral gene transfer using ultrasound: enhancement of transfection efficiency of naked plasmid DNA in skeletal muscle," Gene Ther. 9, 372-380 (2002).

5. U. K. Tirlapur and K. König, "Targeted transfection by femtosecond laser," Nature (London) 418, 290-291 (2002).

6. M. Ogura, S. Sato, K. Nakanishi, M. Uenoyama, T. Kiyozumi, D Saitoh, T. Ikeda, H. Ashida, and M. Obara, "In vivo targeted gene transfer in skin by the use of laser-induced stress waves," Lasers Surg. Med. 34, 242-248 (2004).

7. M. Terakawa, M. Ogura, S. Sato, H. Wakisaka, H. Ashida, M. Uenoyama, Y. Masaki, and M. Obara, "Gene transfer into mammalian cells by use of a nanosecond pulsed laser-induced stress wave," Opt. Lett. 29, 1227-1229 (2004). Erratum in: Opt. Lett. 29, 2190 (2004).

8. D. Goula, J. S. Remy, P. Erbacher, M. Wasowicz, G. Levi, B. Abdallah, and B. A. Demeneix, "Size, diffusibility and transfection performance of linear PEI/DNA complexes in the mouse central nervous system," Gene Ther. 5, 712-717 (1998).

9. Y. Kuroki, K. Fukushima, Y. Kanda, K. Mizuno, and Y. Watanabe, "Neuroprotection by estrogen via extracellular signal-regulated kinase against quinolinic acid-induced cell death in the rat hippocampus," Eur. J. Neurosci. 13, 472-476 (2001). 